\title{
Adaptive rational block Arnoldi methods for model reductions in large-scale MIMO dynamical systems
}

\author{
Oussama Abidi ${ }^{1}$, Mustapha Hached ${ }^{2}$ and Khalide Jbilou ${ }^{1}$ \\ ${ }^{1}$ L.M.P.A. Universite du Littoral, 50 rue F. Buisson, BP 699, F-62228 Calais Cedex France \\ ${ }^{2}$ Laboratoire Painlevé UMR 8524 (ANO-EDP), UFR Mathématiques, Université des Sciences et Technologies de Lille, IUT A \\ Dpartement Chimie, Rue de la Recherche (lieu-dit Le Recueil), BP 179 - 59653 Villeneuve d’ Ascq Cedex, France
}

Received: 17 April 2016, Revised: 27 April 2016, Accepted: 28 April 2016

Published online: 28 April 2016.

\begin{abstract}
In recent years, a great interest has been shown towards Krylov subspace techniques applied to model order reduction of large-scale dynamical systems. A special interest has been devoted to single-input single-output (SISO) systems by using moment matching techniques based on Arnoldi or Lanczos algorithms. In this paper, we consider multiple-input multiple-output (MIMO) dynamical systems and introduce the rational block Arnoldi process to design low order dynamical systems that are close in some sense to the original MIMO dynamical system. Rational Krylov subspace methods are based on the choice of suitable shifts that are selected a priori or adaptively. In this paper, we propose an adaptive selection of those shifts and show the efficiency of this approach in our numerical tests. We also give some new block Arnoldi-like relations that are used to propose an upper bound for the norm of the error on the transfer function.
\end{abstract}

Keywords: Dynamical systems, model reduction, rational block Krylov subspaces, transfer functions.

\section{Introduction}

Let us consider a linear time-invariant (LTI) multi-input and multi-output (MIMO) system described by the state-space equations

$$
\left\{\begin{array}{l}
\dot{x}(t)=A x(t)+B u(t) \\
y(t)=C x(t),
\end{array}\right.
$$

where $x(t) \in \mathbb{R}^{n}$ denotes the state vector and $u(t), y(t) \in \mathbb{R}^{p}$ are the input and output vectors respectively of the (LTI) system (1). The matrix $A \in \mathbb{R}^{n \times n}$ is assumed to be large and sparse, and $B, C^{T} \in \mathbb{R}^{n \times p}$ are tall matrices with $p \ll n$. For single-input single-output (SISO) systems, the matrices $B$ and $C$ are vectors (i.e $p=1$ ).

The linear time invariant system (1) arises in simulations of dynamical systems where partial differential equations are involved and matrices $A$ and $B$ which are generated by the discretization of these equations are often very large. In many cases, the large state-space dimension (or order) $n$ of the system (1) makes the simulations very difficult. Therefore, it is necessary to seek for a lower order model whose behaviour is close to the original:

$$
\left\{\begin{array}{l}
\dot{x}_{m}(t)=A_{m} x_{m}(t)+B_{m} u(t) \\
y_{m}(t)=C_{m} x_{m}(t)
\end{array}\right.
$$

such that $A_{m} \in \mathbb{R}^{m \times m}, B_{m}, C_{m}^{T} \in \mathbb{R}^{m \times p}, x_{m}(t), y_{m}(t) \in \mathbb{R}^{m}$, and $m \ll n$, while maintaining the most relevant properties of the original system (1). 
Many existing model order reduction methods such as Padé approximation [11,28], balanced truncation [21], optimal Hankel norm $[9,10]$ and Krylov subspace based methods In particular the Arnoldi algorithm $[4,5,18,19]$ takes advantage of the sparsity of the large-scale model and has been extensively used for large problems; see $[1,18,15]$. When using block Krylov subspaces, one projects the system matrices of the original problem onto the subspace $\mathscr{K}_{m}(A, B)=$ Range $\left\{B, A B, \ldots, A^{m-1} B\right\}$ generated by the columns of the matrices $B, A B, \ldots, A^{m-1} B$ and try to get a sufficiently accurate reduced system with a moderate space dimension.

In this work, we will consider the rational block Krylov subspace which is a subspace of $\mathbb{R}^{n}$ generated by the columns of the matrices $B,\left(A-s_{2} I\right)^{-1} B, \ldots, \prod_{i=2}^{m}\left(A-s_{i} I\right)^{-1} B$, where $s_{2}, \ldots, s_{m}$ are some selected complex shifts. The original large problem is projected onto this block Krylov subspace to get a new low order dynamical system that is close in some sense to initial one. The rational Krylov subspace procedure was originally proposed by Ruhe [26] in the context of approximating interior eigenvalues and have been used during the last years for model order reduction; see [11]. The selection of good shifts is a crucial issue for the quality of the approximation. The use of rational Krylov spaces is recognized as a powerful tool within model order reduction techniques for linear dynamical systems, however its success has been hindered by the lack of a parameter-free procedure, which would effectively generate the sequence of shifts used to build the space. Major efforts have been devoted to this question in the recent years; see for example [6,7,8,18, 20,22]. In the context of $\mathscr{H}_{2}$-optimality reduction, an interesting attempt to provide an automatic selection has been recently proposed in [13]. However, the computational and memory costs of this approach have not been fully assessed. We also mention the early contribution due to Grimme [11] for determining a sequence of shifts. Another approach has been recently developed in [6] to generate these parameters. In this paper, we propose an adaptive computation of the shifts for building the rational space by minimizing, at each iteration of the process, some matrix norms. We will derive some theoretical results such as upper bounds for the norm of the error on the transfer function. Some numerical tests will be provided in order to compare our approach with other existing methods.

The paper is organized as follow: In Section 2, we introduce the rational block Arnoldi and give some new algebraic relations. Section 3 is devoted to the selection of the shifts that are used in the construction of rational Krylov subspaces and we give an error bound for the norm of the error on the transfer function. A new modified rational block Arnoldi is proposed in Section 4 and some new Arnoldi-like relations are proposed. The last section is devoted to some numerical tests and comparisons to some well known model order reduction methods.

We will use the following notations: the 2-norm of a vector or of a matrix will be denoted by $\|\cdot\|$ and $I_{p}$ is the identity matrix of dimension $p \times p$.

\section{The rational block Arnoldi method}

In this section we will describe the rational block Arnoldi algorithm for computing an orthonormal basis of the rational block Krylov subspace defined for a given matrix $B \in \mathbb{R}^{n \times p}$ as

$$
\mathbf{K}_{m}(A, B)=\operatorname{Range}\left(\left\{B,\left(A-s_{2} I\right)^{-1} B, \ldots, \prod_{i=2}^{m}\left(A-s_{i} I\right)^{-1} B\right\}\right) .
$$

The rational block Arnoldi algorithm generates a sequence of $n \times p$ blocks $\left\{V_{1}, \ldots, V_{m}\right\}$ whose columns form an orthonormal basis of the rational block Krylov subspace $\mathbf{K}_{m}(A, B)$. The algorithm is described as follows 


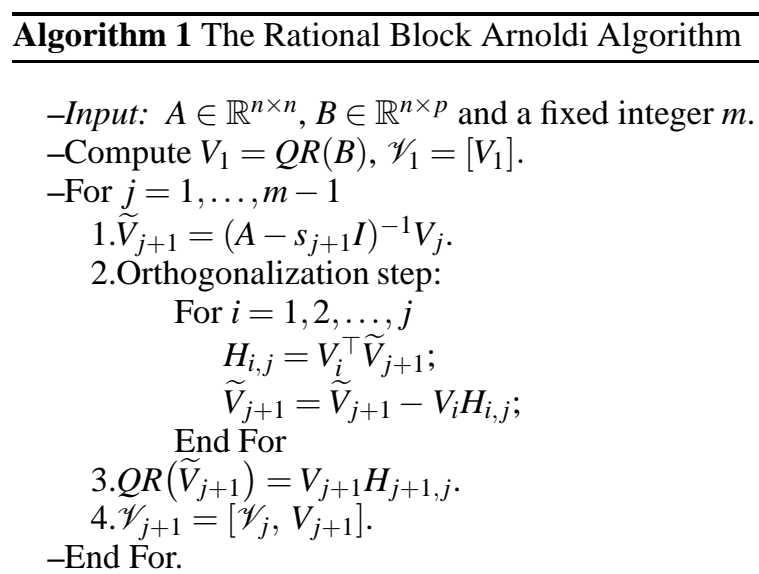

The shifts $s_{2}, \ldots, s_{m}$ will be chosen a priori or a posteriori during the process and this will be explained later. After $m$ steps, the rational block Arnoldi algorithm generates a block matrix $\mathscr{V}_{m}=\left[V_{1}, \ldots, V_{m}\right] \in \mathbb{R}^{n \times m p}$ whose columns form an orthonormal basis of the rational block Krylov subspace $\mathbf{K}_{m}(A, B)$ and an upper $(m+1) p \times m p$ block Hessenberg matrix $\overline{\mathscr{H}}_{m}$ whose blocks $H_{i, j}$ are defined by Algorithm 1 . The $m p \times m p$ upper block Hessenberg matrix $\mathscr{H}_{m}$ is obtained from $\overline{\mathscr{H}}_{m}$ by deleting its last $p$-rows. In the sequel we will also use the restriction matrix $\mathscr{T}_{m}$ defined by $\mathscr{T}_{m}:=\mathscr{V}_{m}^{*} A \mathscr{V}_{m}$. We first give some new algebraic relations generalising the well known Arnoldi like relation given for the classical case.

Proposition 1. Let $\mathscr{V}_{m}, \overline{\mathscr{H}}_{m}$ and $\mathscr{H}_{m}$ be the matrices generated by the rational block Arnoldi algorithm and let $\mathscr{S}_{m}$ be the block-diagonal matrix blkdiag $\left(s_{2} I_{p}, \ldots, s_{m+1} I_{p}\right)$ where $\left\{s_{2}, \ldots, s_{m+1}\right\}$ denotes the set of shifts used in the algorithm. Then we have the following relation

$$
\mathscr{T}_{m}:=\mathscr{V}_{m}^{*} A \mathscr{V}_{m}=\left(I_{m p}+\mathscr{H}_{m} S_{m}-\mathscr{V}_{m}^{*} A V_{m+1} H_{m+1, m} E_{m}^{*}\right) \mathscr{H}_{m}^{-1}
$$

where $E_{m}^{*}=\left[0_{p}, \ldots, 0_{p}, I_{p}\right]=\left(e_{m}^{*} \otimes I_{p}\right)$.

Proof. After $m$ steps of the rational block Arnoldi algorithm, we have

$$
\left(A-s_{j+1} I_{n}\right)^{-1} V_{j}=\sum_{i=1}^{j+1} V_{i} H_{i, j} \quad \text { for } j=1, \ldots, m
$$

then

$$
V_{j}=A\left(\sum_{i=1}^{j+1} V_{i} H_{i, j}\right)-s_{j+1}\left(\sum_{i=1}^{j+1} V_{i} H_{i, j}\right) \quad \text { for } j=1, \ldots, m .
$$

This gives the following relation

$$
\mathscr{V}_{m}=A\left(\mathscr{V}_{m+1} \overline{\mathscr{H}}_{m}\right)-\left(\mathscr{V}_{m+1} \overline{\mathscr{H}}_{m}\right) \mathscr{S}_{m}
$$

which can also be written as

$$
\mathscr{V}_{m}=A\left(\mathscr{V}_{m} \mathscr{H}_{m}+V_{m+1} H_{m+1, m} E_{m}^{*}\right)-\left(\mathscr{V}_{m} \mathscr{H}_{m}+V_{m+1} H_{m+1, m} E_{m}^{*}\right) \mathscr{S}_{m}
$$

Multiplying the last equality on the left by $\mathscr{V}_{m}^{*}$ and using the fact that the blocks $V_{1}, \ldots, V_{m+1}$ are orthonormal, we get the identity

$$
I_{m p}=\mathscr{T}_{m} \mathscr{H}_{m}+\mathscr{V}_{m}^{*} A V_{m+1} H_{m+1, m} E_{m}^{*}-\mathscr{H}_{m} \mathscr{S}_{m}
$$


Finally, we can deduce the relation

$$
\mathscr{T}_{m}=\left(I_{m p}+\mathscr{H}_{m} \mathscr{S}_{m}-\mathscr{V}_{m}^{*} A V_{m+1} H_{m+1, m} E_{m}^{*}\right) \mathscr{H}_{m}^{-1}
$$

which ends the proof.

We can also state the following result

Proposition 2. Under the same assumptions as in Proposition 1, we have

$$
A \mathscr{V}_{m}=\mathscr{V}_{m} \mathscr{T}_{m}-\left(I_{n}-\mathscr{V}_{m} \mathscr{V}_{m}^{*}\right) A V_{m+1} H_{m+1, m} E_{m}^{*} \mathscr{H}_{m}^{-1}+V_{m+1} H_{m+1, m} E_{m}^{*} S_{m} \mathscr{H}_{m}^{-1}
$$

Proof. As stated in the previous proposition, we have

$$
\mathscr{V}_{m}=A\left(\mathscr{V}_{m} \mathscr{H}_{m}+V_{m+1} H_{m+1, m} E_{m}^{*}\right)-\left(\mathscr{V}_{m} \mathscr{H}_{m}+V_{m+1} H_{m+1, m} E_{m}^{*}\right) \mathscr{S}_{m}
$$

Hence, we can write

$$
\begin{aligned}
A \mathscr{V}_{m} \mathscr{H}_{m} & =\mathscr{V}_{m}-A V_{m+1} H_{m+1, m} E_{m}^{*}+\mathscr{V}_{m} \mathscr{H}_{m} \mathscr{S}_{m}+V_{m+1} H_{m+1, m} E_{m}^{*} \mathscr{S}_{m} \\
& =\mathscr{V}_{m}\left(I_{m p}+\mathscr{H}_{m} \mathscr{S}_{m}\right)-A V_{m+1} H_{m+1, m} E_{m}^{*}+V_{m+1} H_{m+1, m} E_{m}^{*} \mathscr{S}_{m}
\end{aligned}
$$

Using Proposition 1, we obtain the following relation

$$
\begin{aligned}
A \mathscr{V}_{m} \mathscr{H}_{m} & =\mathscr{V}_{m}\left(\mathscr{T}_{m} \mathscr{H}_{m}+\mathscr{V}_{m}^{*} A V_{m+1} H_{m+1, m} E_{m}^{*}\right)-A V_{m+1} H_{m+1, m} E_{m}^{*}+V_{m+1} H_{m+1, m} E_{m}^{*} \mathscr{S}_{m} \\
& =\mathscr{V}_{m} \mathscr{T}_{m} \mathscr{H}_{m}-\left(I_{n}-\mathscr{V}_{m} \mathscr{V}_{m}^{*}\right) A V_{m+1} H_{m+1, m} E_{m}^{*}+V_{m+1} H_{m+1, m} E_{m}^{*} \mathscr{S}_{m}
\end{aligned}
$$

Therefore

$$
A \mathscr{V}_{m}=\mathscr{V}_{m} \mathscr{T}_{m}-\left(I_{n}-\mathscr{V}_{m} \mathscr{V}_{m}^{*}\right) A V_{m+1} H_{m+1, m} E_{m}^{*} \mathscr{H}_{m}^{-1}+V_{m+1} H_{m+1, m} E_{m}^{*} \mathscr{S}_{m} \mathscr{H}_{m}^{-1}
$$

\section{An adaptive computation of the shifts}

In this section, we will see some a posteriori and a priori procedures for selecting good shifts used during the construction of the rational block Arnoldi bases. This is a crucial problem when using rational Krylov subspace methods.

\subsection{An a priori selection of the shifts}

We briefly describe an a priori way for selecting the complex shifts. This technique was introduced by Penzl [25] and implemented in the routine lp_para of the library LYAPACK [23]. The parameters are selected by solving the following min-max problem; see $[25,30,31]$ for more details.

$$
\left\{s_{1}, s_{2}, \ldots, s_{l}\right\}=\arg \min _{\left\{\mu_{1}, \mu_{2}, \ldots, \mu_{l}\right\} \in \mathbb{C}_{-}}\left(\max _{\lambda \in \sigma(A)} \frac{\left|\left(\lambda-\mu_{1}\right) \ldots\left(\lambda-\mu_{l}\right)\right|}{\left|\left(\lambda+\mu_{1}\right) \ldots\left(\lambda+\mu_{l}\right)\right|}\right),
$$

where $\sigma(A)$ denotes the spectrum of the matrix $A$.

As we generally are unable to compute the spectrum of matrix $A$, the classical approach is to cover it by a domain 
$\Omega \subset \mathbb{C}_{-}$and then to solve the minimax problem with respect to $\Omega$. In [24,25], a heuristic procedure was proposed to find "sub-optimal" parameters. This technique first generates a discrete set which approximates the spectrum $\sigma(A)$ using a pair of Arnoldi processes. The first one acts on the matrix $A$ and generates $k_{+}$Ritz values which tend to approximate the eigenvalues far from the origin. The second process, acting on the matrix $A^{-1}$, generates $k_{-}$Ritz values whose inverses are close to the origin. The set of shift parameters is then chosen as a subset of these Ritz values. This procedure is widely used in the ADI-type methods for solving large scale matrix equations such as Lyapunov or Sylvester matrix equations; see for example $[3,16]$

\subsection{A new adaptive selection of the shifts}

In this subsection we propose an adaptive technique for computing the shifts that are used to build the rational Krylov subspace. This procedure automatically generates the sequence of shifts during the construction of the rational Arnoldi subspaces.

A classical way of relating the input to output is to use the transfer function (or impulse response in the time domain) of the LTI system (1). Indeed, applying the Laplace transform

$$
\mathscr{L}(f)(s):=\int_{0}^{\infty} e^{-s t} f(t) d t
$$

to the dynamical system (1), we obtain

$$
\left\{\begin{array}{l}
s X(s)=A X(s)+B U(s) \\
Y(s)=C X(s)
\end{array},\right.
$$

where $X(s), Y(s)$ and $U(s)$ are the Laplace transforms of $x(t), y(t)$ and $u(t)$, respectively. Eliminating $X(s)$ in the previous two equations, we get

$$
Y(s)=H(s) U(s)
$$

where

$$
H(s)=C\left(s I_{n}-A\right)^{-1} B
$$

The rational function $H(s)$ is called the transfer function of the system (1). We recall that most model order reduction techniques, for example the moment-matching approaches, are based on the approximation of this transfer function; see $[2,12,18]$. If the number of state variables is very large, it would be very difficult to use the full system for simulation or run-on-time control. So it is reasonable to look for lower order models that approximate the behavior of the original models. This will be done by approximating the transfer function (5).

Let us write $H(s)=C X$ where $X \in \mathbb{R}^{n \times p}$ is the solution of the matrix linear system

$$
\left(s I_{n}-A\right) X=B
$$

In order to approximate the transfer function $H$, we will look for approximations of the solution $X$ of the multiple second member linear system (6). Let $X_{m}$ denotes the approximate solution obtained by the Galerkin projection method onto the rational Krylov subspace $\mathbf{K}_{m}(A, B)$. This approximate solution is given by

$$
X_{m}=\mathscr{V}_{m}\left(s I_{m p}-\mathscr{T}_{m}\right)^{-1} \mathscr{V}_{m}^{*} B
$$


where $\mathscr{T}_{m}=\mathscr{V}_{m}^{*} A \mathscr{V}_{m}$, hence the transfer function $H$ is approximated by the low order transfer function corresponding to the projected low order dynamical system and given by

$$
H_{m}(s)=C \mathscr{V}_{m}\left(s I_{m p}-\mathscr{T}_{m}\right)^{-1} \mathscr{V}_{m}^{*} B
$$

which can be written as

$$
H_{m}(s)=C_{m}\left(s I_{m p}-\mathscr{T}_{m}\right)^{-1} B_{m}
$$

where $C_{m}=C \mathscr{V}_{m}$ and $B_{m}=\mathscr{V}_{m}^{*} B$

In the sequel, we will give an expression for the norm of the error $H(s)-H_{m}(s)$, which will be used for the selection of our shift parameters. First, we recall the norm $\mathscr{H}_{\infty}$ for a matrix-valued function

$$
\|H\|_{\infty}=\sup _{y \in \mathbb{R}} \sigma_{\max }(H(i y))
$$

Indeed we have:

$$
\begin{aligned}
H(s)-H_{m}(s) & =C X-C X_{m} \\
& =C\left(s I_{n}-A\right)^{-1} B-C X_{m} \\
& =C\left(s I_{n}-A\right)^{-1}\left[B-\left(s I_{n}-A\right) X_{m}\right]
\end{aligned}
$$

By applying the norm described above, we obtain

$$
\left\|H(s)-H_{m}(s)\right\| \leq\left\|C\left(s I_{n}-A\right)^{-1}\right\|\left\|\Gamma_{m}\right\|_{\infty}
$$

where $\Gamma_{m}=B-\left(s I_{n}-A\right) X_{m}$. So, one way for selecting a new shift, is to choose those that allows us to reach $\left\|\Gamma_{m}\right\|_{\infty}$. Hence, our new shift $s_{m+1}$ will be chosen as

$$
s_{m+1}=\left\{s \in \mathbb{R}: \sigma_{\max }\left(\Gamma_{m}(i s)\right)=\left\|\Gamma_{m}\right\|_{\infty}\right\}
$$

As we will see in the numerical tests, this simple procedure gives good results.

\subsection{An error expression for the transfer function}

In the following proposition we give an upper bound for the 2-norm of the error $H(s)-H_{m}(s)$.

Proposition 3. Let $H$ be the transfer function defined in (5) and let $H_{m}$ be its approximation. Then, under the conditions $\|A\|<|s|$, we have the follwing upper bound:

$$
H(s)-H_{m}(s)=C\left(s I_{n}-A\right)^{-1}\left[-\left(I_{n}-\mathscr{V}_{m} \mathscr{V}_{m}^{*}\right) A V_{m+1} H_{m+1, m} E_{m}^{*} \mathscr{H}_{m}^{-1}+V_{m+1} H_{m+1, m} E_{m}^{*} S_{m} \mathscr{H}_{m}^{-1}\right]\left(s I_{m p}-\mathscr{T}_{m}\right)^{-1} \mathscr{V}_{m}^{*} B
$$

And

$$
\left\|H(s)-H_{m}(s)\right\| \leq \frac{\|C\|\left\|H_{m+1, m}\right\|\left(\|A\|+\left\|S_{m}\right\|\right)\left\|\mathscr{H}_{m}^{-1}\right\|}{(|s|-\|A\|)}\left\|\left(s I_{m p}-\mathscr{T}_{m}\right)^{-1} \mathscr{V}_{m}^{*} B\right\|
$$


Proof. We have:

$$
\begin{aligned}
H(s)-H_{m}(s) & =C\left(s I_{n}-A\right)^{-1} B-C_{m}\left(s I_{m p}-\mathscr{T}_{m}\right)^{-1} B_{m} \\
& =C\left(s I_{n}-A\right)^{-1} B-C \mathscr{V}_{m}\left(s I_{m p}-\mathscr{T}_{m}\right)^{-1} \mathscr{V}_{m}^{*} B \\
& =C\left(s I_{n}-A\right)^{-1}\left[B-\left(s I_{n}-A\right) \mathscr{V}_{m}\left(s I_{m p}-\mathscr{T}_{m}\right)^{-1} \mathscr{V}_{m}^{*} B\right] \\
& =C\left(s I_{n}-A\right)^{-1}\left[B-\left(s \mathscr{V}_{m}-A \mathscr{V}_{m}\right)\left(s I_{m p}-\mathscr{T}_{m}\right)^{-1} \mathscr{V}_{m}^{*} B\right]
\end{aligned}
$$

Using Proposition 2, we obtain

$$
\begin{aligned}
H(s)-H_{m}(s) & =C\left(s I_{n}-A\right)^{-1}\left[B-\left(s \mathscr{V}_{m}-\mathscr{V}_{m} \mathscr{T}_{m}+\left(I_{n}-\mathscr{V}_{m} \mathscr{V}_{m}^{*}\right) A V_{m+1} H_{m+1, m} E_{m}^{*} \mathscr{H}_{m}^{-1}\right.\right. \\
& \left.\left.-V_{m+1} H_{m+1, m} E_{m}^{*} S_{m} \mathscr{H}_{m}^{-1}\right)\left(s I_{m p}-\mathscr{T}_{m}\right)^{-1} \mathscr{V}_{m}^{*} B\right] \\
& =C\left(s I_{n}-A\right)^{-1}\left[B-\left(s \mathscr{V}_{m}-\mathscr{V}_{m} \mathscr{T}_{m}\right)\left(s I_{m p}-\mathscr{T}_{m}\right)^{-1} \mathscr{V}_{m}^{*} B-\left(\left(I_{n}-\mathscr{V}_{m} \mathscr{V}_{m}^{*}\right)\right.\right. \\
& \left.\left.\times A V_{m+1} H_{m+1, m} E_{m}^{*} \mathscr{H}_{m}^{-1}-V_{m+1} H_{m+1, m} E_{m}^{*} S_{m} \mathscr{H}_{m}^{-1}\right)\left(s I_{m p}-\mathscr{T}_{m}\right)^{-1} \mathscr{V}_{m}^{*} B\right] \\
& =C\left(s I_{n}-A\right)^{-1}\left[B-\mathscr{V}_{m} \mathscr{V}_{m}^{*} B-\left(\left(I_{n}-\mathscr{V}_{m} \mathscr{V}_{m}^{*}\right) A V_{m+1} H_{m+1, m} E_{m}^{*} \mathscr{H}_{m}^{-1}\right.\right. \\
& \left.\left.-V_{m+1} H_{m+1, m} E_{m}^{*} S_{m} \mathscr{H}_{m}^{-1}\right)\left(s I_{m p}-\mathscr{T}_{m}\right)^{-1} \mathscr{V}_{m}^{*} B\right] .
\end{aligned}
$$

As $B$ is in the rational Krylov subspace (3), then we have $\mathscr{V}_{m} \mathscr{V}_{m}^{*} B=B$. This gives the following expression

$$
\begin{aligned}
H(s)-H_{m}(s)=C\left(s I_{n}-A\right)^{-1}[ & -\left(I_{n}-\mathscr{V}_{m} \mathscr{V}_{m}^{*}\right) A V_{m+1} H_{m+1, m} E_{m}^{*} \mathscr{H}_{m}^{-1} \\
& \left.+V_{m+1} H_{m+1, m} E_{m}^{*} S_{m} \mathscr{H}_{m}^{-1}\right]\left(s I_{m p}-\mathscr{T}_{m}\right)^{-1} \mathscr{V}_{m}^{*} B .
\end{aligned}
$$

By applying the 2-norm we obtain

$$
\begin{aligned}
\left\|H(s)-H_{m}(s)\right\| & \leq\left\|C\left(s I_{n}-A\right)^{-1}\right\|\left[\left\|\left(I_{n}-\mathscr{V}_{m} \mathscr{V}_{m}^{*}\right) A V_{m+1} H_{m+1, m} E_{m}^{*} \mathscr{H}_{m}^{-1}\right\|\right. \\
& \left.+\left\|V_{m+1} H_{m+1, m} E_{m}^{*} S_{m} \mathscr{H}_{m}^{-1}\right\|\right] \times\left\|\left(s I_{m p}-\mathscr{T}_{m}\right)^{-1} \mathscr{V}_{m}^{*} B\right\|
\end{aligned}
$$

Therefore, as $\|A\|<|s|$ we obtain

$$
\begin{aligned}
\left\|H(s)-H_{m}(s)\right\| & \leq \frac{\|C\|}{(|s|-\|A\|)}\left[\left\|\left(I_{n}-\mathscr{V}_{m} \mathscr{V}_{m}^{*}\right) A V_{m+1} H_{m+1, m} E_{m}^{*} \mathscr{H}_{m}^{-1}\right\|\right. \\
& \left.+\left\|V_{m+1} H_{m+1, m} E_{m}^{*} S_{m} \mathscr{H}_{m}^{-1}\right\|\right]\left\|\left(s I_{m p}-\mathscr{T}_{m}\right)^{-1} \mathscr{V}_{m}^{*} B\right\|
\end{aligned}
$$

furthermore as $I_{n}-\mathscr{V}_{m} \mathscr{V}_{m}^{*}$ is an orthogonal projection and $\left\|V_{m+1}\right\|=1$, we get

$$
\left\|H(s)-H_{m}(s)\right\| \leq \frac{\|C\|\left\|H_{m+1, m}\right\|\left(\|A\|+\left\|S_{m}\right\|\right)\left\|\mathscr{H}_{m}^{-1}\right\|}{(|s|-\|A\|)}\left\|\left(s I_{m p}-\mathscr{T}_{m}\right)^{-1} \mathscr{V}_{m}^{*} B\right\| .
$$

\section{A modified rational block Arnoldi algorithm}

In this section, we describe a generalization of the rational Krylov subspace, allowing some shifts to be equal to infinity. At each step $j+1$, the algorithm computes a new block $\widetilde{V}_{j+1}=\left(A-s_{j+1} I\right)^{-1} V_{j}$ if $s_{j+1}$ is finite and $\widetilde{V}_{j+1}=A V_{j}$ if $s_{j+1}=\infty$. The modified rational Arnoldi algorithm is summarized as follows 


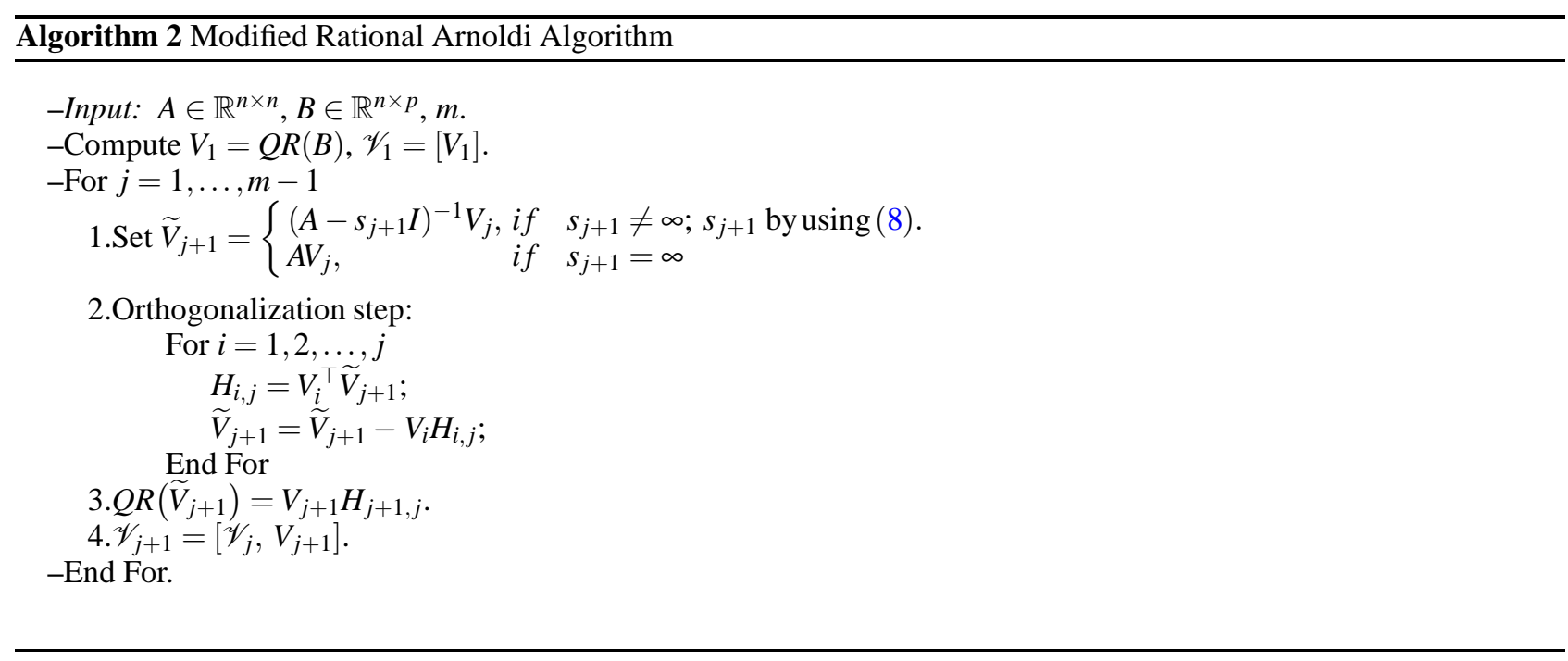

The idea of including infinity as a possible interpolation point could be considered as a generalization of the extended block Arnoldi algorithm [14,29]. This new version also allows one to obtain new simple Arnoldi-like relations that could be used when deriving for example error bounds or residual error expressions and perturbation analysis. Using the modified rational Arnoldi algorithm, we can state the following simple Arnoldi-like relations

Proposition 4. Let $\mathscr{S}=\left\{s_{2}, \ldots, s_{m}\right\} \subset \mathbb{C}$ and $\mathscr{V}_{m}=\left[V_{1}, \ldots, V_{m+1}\right] \in \mathbb{R}^{n \times(m+1) p}$ as generated by running Algorithm 2 for one extra interpolation point at $s_{m+1}=\infty$. Then the following Arnoldi-like equations are satisfied

$$
\begin{aligned}
A \mathscr{V}_{m} & =\mathscr{V}_{m+1} \overline{\mathscr{T}}_{m} \\
& =\mathscr{V}_{m} \mathscr{T}_{m}+V_{m+1} N_{m},
\end{aligned}
$$

where $\overline{\mathscr{T}}_{m}=\mathscr{V}_{m+1}^{*} A \mathscr{V}_{m}, \mathscr{T}_{m}=\mathscr{V}_{m}^{*} A \mathscr{V}_{m}$ and $N_{m}=V_{m+1}^{*} A \mathscr{V}_{m}$

Proof. From Algorithm 2, it easy to see that the following relations are satisfied

$$
\operatorname{Range}\left(\left[\mathscr{V}_{m} A V_{m}\right]\right)=\operatorname{Range}\left(\mathscr{V}_{m+1}\right), \quad \text { and } \mathscr{V}_{m+1}^{*} \mathscr{V}_{m+1}=I_{(m+1) p}
$$

Let us now prove that

$$
\operatorname{Range}\left(A \mathscr{V}_{m}\right) \subset \operatorname{Range}\left(\mathscr{V}_{m+1}\right) .
$$

Indeed, after $m-1$ iterations of the rational Arnoldi algorithm, the proof of Proposition 1 gives us

$$
\mathscr{V}_{m-1}=A\left(\mathscr{V}_{m-1} \mathscr{H}_{m-1}+V_{m} H_{m, m-1} E_{m-1}^{*}\right)-\left(\mathscr{V}_{m-1} \mathscr{H}_{m-1}+V_{m} H_{m, m-1} E_{m-1}^{*}\right) \mathscr{S}_{m-1},
$$

then

$$
A \mathscr{V}_{m-1}=\mathscr{V}_{m-1} \mathscr{H}_{m-1}^{-1}-A V_{m} H_{m, m-1} E_{m-1}^{*} \mathscr{H}_{m-1}^{-1}+\left(\mathscr{V}_{m-1} \mathscr{H}_{m-1}+V_{m} H_{m, m-1} E_{m-1}^{*}\right) \mathscr{S}_{m-1} \mathscr{H}_{m-1}^{-1}
$$

Using the fact that $\operatorname{Range}\left(A V_{m}\right) \subset \operatorname{Range}\left(\mathscr{V}_{m+1}\right)$, it is clear that $\operatorname{Range}\left(A \mathscr{V}_{m-1}\right) \subset \operatorname{Range}\left(\mathscr{V}_{m+1}\right)$, and $\operatorname{Range}\left(A \mathscr{V}_{m}\right) \subset$ Range $\left(\mathscr{V}_{m+1}\right)$. Therefore we have

$$
A \mathscr{V}_{m}=\mathscr{V}_{m+1} \overline{\mathscr{T}}_{m}
$$


for some matrix $\overline{\mathscr{T}}_{m}$. Since $\mathscr{V}_{m+1}$ is orthonormal, multiplying (9) on the left by $\mathscr{V}_{m+1}^{*}$, we get $\overline{\mathscr{T}}_{m}=\mathscr{V}_{m+1}^{*} A \mathscr{V}_{m}$. We can also see that

$$
A \mathscr{V}_{m}=\mathscr{V}_{m} \mathscr{L}_{m}+V_{m+1} N_{m}
$$

for some matrices $\mathscr{L}_{m}$ and $N_{m}$. Therefore, multiplying (10) on the left by $\mathscr{V}_{m}^{*}$ gives

$$
\mathscr{L}_{m}=\mathscr{T}_{m}=\mathscr{V}_{m}^{*} A \mathscr{V}_{m},
$$

and multiplying (10) by $V_{m+1}^{*}$ we get

$$
N_{m}=V_{m+1}^{*} A \mathscr{V}_{m}
$$

This completes the proof.

In the next proposition, we give a new expression of the error $H(s)-H_{m}(s)$ which could be used to compute a new upper bound for the norm of the error on the transfer function.

Proposition 5. Under the hypothesis of Proposition 4, we have the following relation

$$
H(s)-H_{m}(s)=C\left(s I_{n}-A\right)^{-1} V_{m+1} N_{m}\left(s I_{m p}-\mathscr{T}_{m}\right)^{-1} \mathscr{V}_{m}^{*} B,
$$

and we also have the upper bounds for the norm of the error given by

$$
\begin{aligned}
\left\|H(s)-H_{m}(s)\right\| & \leq \frac{\|C\|}{|s|-\|A\|}\left\|N_{m}\right\|\left\|\left(s I_{m p}-\mathscr{T}_{m}\right)^{-1} \mathscr{V}_{m}^{*} B\right\| \\
& \leq \frac{\|C\|\|B\|\|A\|}{|s|-\|A\|}\left\|\left(s I_{m p}-\mathscr{T}_{m}\right)^{-1}\right\|
\end{aligned}
$$

Proof.

$$
\begin{aligned}
H(s)-H_{m}(s) & =C\left(s I_{n}-A\right)^{-1} B-C_{m}\left(s I_{m p}-\mathscr{T}_{m}\right)^{-1} B_{m} \\
& =C\left(s I_{n}-A\right)^{-1} B-C \mathscr{V}_{m}\left(s I_{m p}-\mathscr{T}_{m}\right)^{-1} \mathscr{V}_{m}^{*} B \\
& =C\left(s I_{n}-A\right)^{-1}\left[B-\left(s I_{n}-A\right) \mathscr{V}_{m}\left(s I_{m p}-\mathscr{T}_{m}\right)^{-1} \mathscr{V}_{m}^{*} B\right] \\
& =C\left(s I_{n}-A\right)^{-1}\left[B-\left(s \mathscr{V}_{m}-A \mathscr{V}_{m}\right)\left(s I_{m p}-\mathscr{T}_{m}\right)^{-1} \mathscr{V}_{m}^{*} B\right] .
\end{aligned}
$$

We use the result of Proposition 4 and we obtain

$$
\begin{aligned}
H(s)-H_{m}(s) & =C\left(s I_{n}-A\right)^{-1}\left[B-\left(s \mathscr{V}_{m}-\mathscr{V}_{m} \mathscr{T}_{m}-V_{m+1} N_{m}\right)\left(s I_{m p}-\mathscr{T}_{m}\right)^{-1} \mathscr{V}_{m}^{*} B\right] \\
& =C\left(s I_{n}-A\right)^{-1}\left[B-\mathscr{V}_{m} \mathscr{V}_{m}^{*} B+V_{m+1} N_{m}\left(s I_{m p}-\mathscr{T}_{m}\right)^{-1} \mathscr{V}_{m}^{*} B\right] .
\end{aligned}
$$

Using the fact that $B$ is in the rational block Krylov subspace $\mathbf{K}_{m}(A, B)$, it follows that

$$
H(s)-H_{m}(s)=C\left(s I_{n}-A\right)^{-1} V_{m+1} N_{m}\left(s I_{m p}-\mathscr{T}_{m}\right)^{-1} \mathscr{V}_{m}^{*} B .
$$

The relations (12) are easily derived from the preceding relation. 


\section{Numerical experiments}

In this section, we give some numerical examples to show the effectiveness of our adaptive rational block Arnoldi method denoted by ARAM when compared to the rational block Arnoldi method RAM with a priori choice of shifts using the Matlab function 1p_para [23], the iterative rational Krylov algorithm IRKA method proposed in [13] and with the method RKSM introduced in [6]. All the experiments were performed on a $1.3 \mathrm{GHz}$ Intel Core i5 laptop with $8 \mathrm{~Gb}$ of RAM. The algorithms were coded in Matlab R2010a. For all the tests, we set $B=C^{\top}=\operatorname{rand}(n, p)$. We used various benchmark matrices as reported in Table 1. We first compared our proposed ARAM method, with the Rational Arnoldi Method RAM for wich we

Table 1: Information for the test problems.

\begin{tabular}{l|l|l|l} 
Matrix A & Size $\mathrm{n}$ & $\|A\|_{F}$ & $\operatorname{cond}(\mathrm{A})$ \\
\hline fdm & 2500 & $2.9996 \mathrm{e}+005$ & $1.0235 \mathrm{e}+003$ \\
fom & 1006 & $1.8283 \mathrm{e}+04$ & 1000 \\
beam & 348 & $5.6430 \mathrm{e}+003$ & $3.7420 \mathrm{e}+007$ \\
CDplayer & 120 & $2.3095 \mathrm{e}+05$ & $1.8149 \mathrm{e}+04$ \\
\hline
\end{tabular}

used an priori choice of shifts calculated by the routine lp_para from [23]. In the first experiment, we considered the fom model and we compared ARAM and the rational block Arnoldi when using the shifts computed via lp_para with $m=8$ and 16.
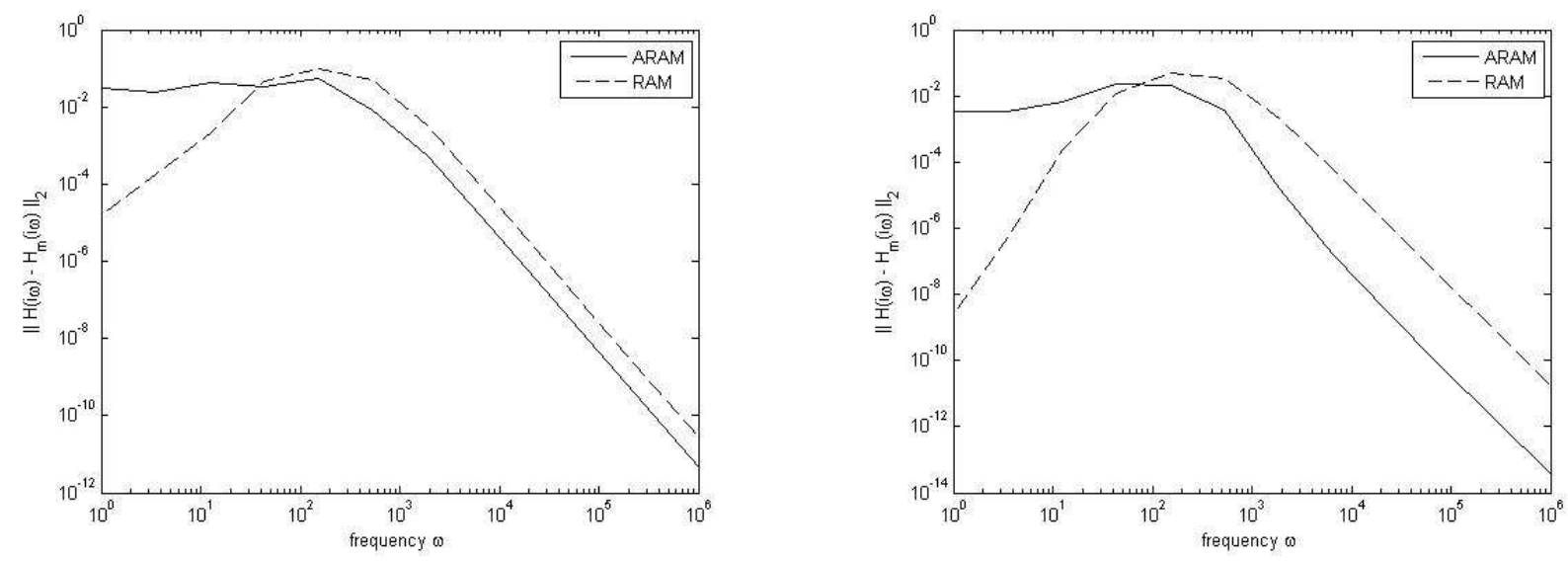

Fig. 1: The fom model: Comparison of ARAM and RAM with lp_para. The error $\sigma_{\max }\left(H(i \omega)-H_{m}(i \omega)\right)$ for $\omega \in\left[1,10^{6}\right]$ with $m=8$ (left) and $m=16$ (right) $(\mathrm{p}=3)$.

In the second experiment, we compared the performances of ARAM and IRKA for the fdm model. In Figure 2, we plotted the curve corresponding to the errors for the norm of the transfer functions for the method IRKA and ARAM. For this experiment, we considered the fdm model from Table 1 where the matrix $A$ is of dimension $n=2500$. The algorithm IRKA starts with a set of parameters chosen randomly as suggested in [13].

We also compared the performance of ARAM to the recent rational Krylov subspace method RKSM developed in [6] for SISO systems $(p=1)$. In this example we consider the CDplayer model. The method RKSM starts with the two input shifts: $s_{0}^{(0)}=10^{-1}$ and $s_{0}^{(1)}=800+i 5.10^{4}$ as suggested in [6] and the obtained results are shown in Figure 3.

For our last experiment, we considered the adaptive rational Arnoldi algorithm with the modified version as described Algorithm 2. This algorithm will be named Modified Adaptive Rational Block Arnoldi Method MARAM. As a test model, 

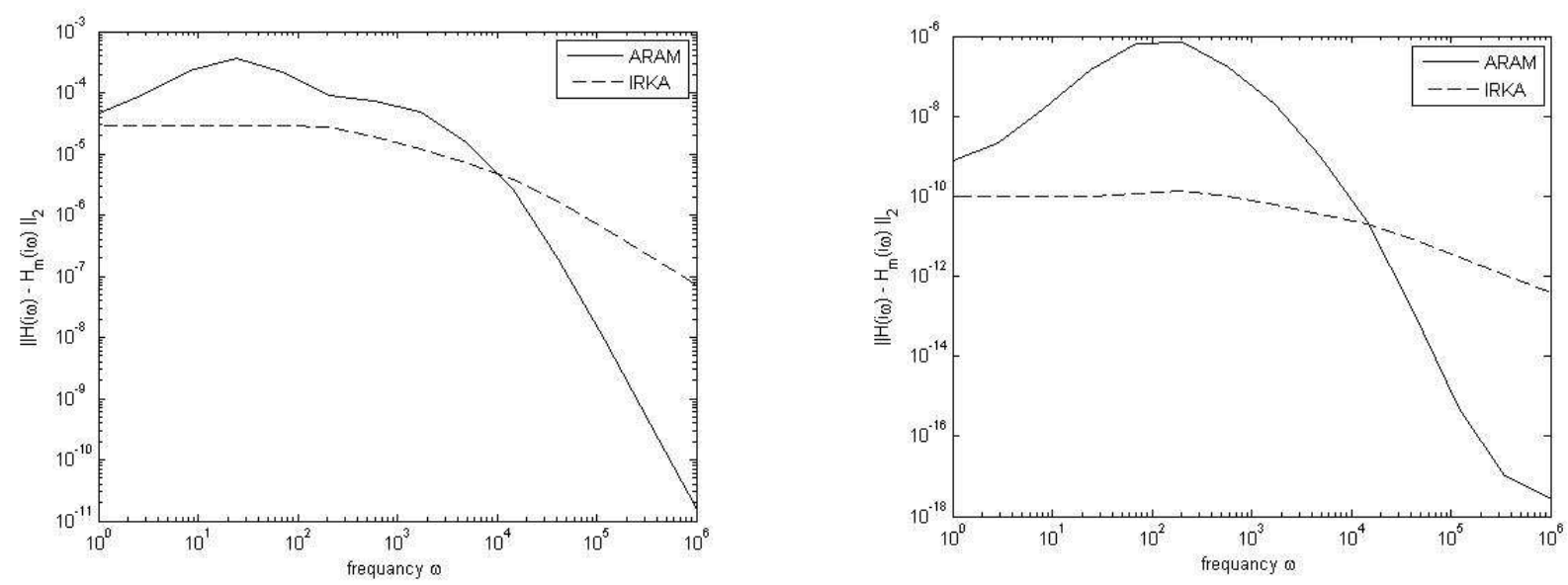

Fig. 2: The fdm model: Comparison of ARAM and IRKA. The error $\sigma_{\max }\left(H(i \omega)-H_{m}(i \omega)\right)$ for $\omega \in\left[1,10^{6}\right]$ with $m=8$ (left) and $m=16$ (right).
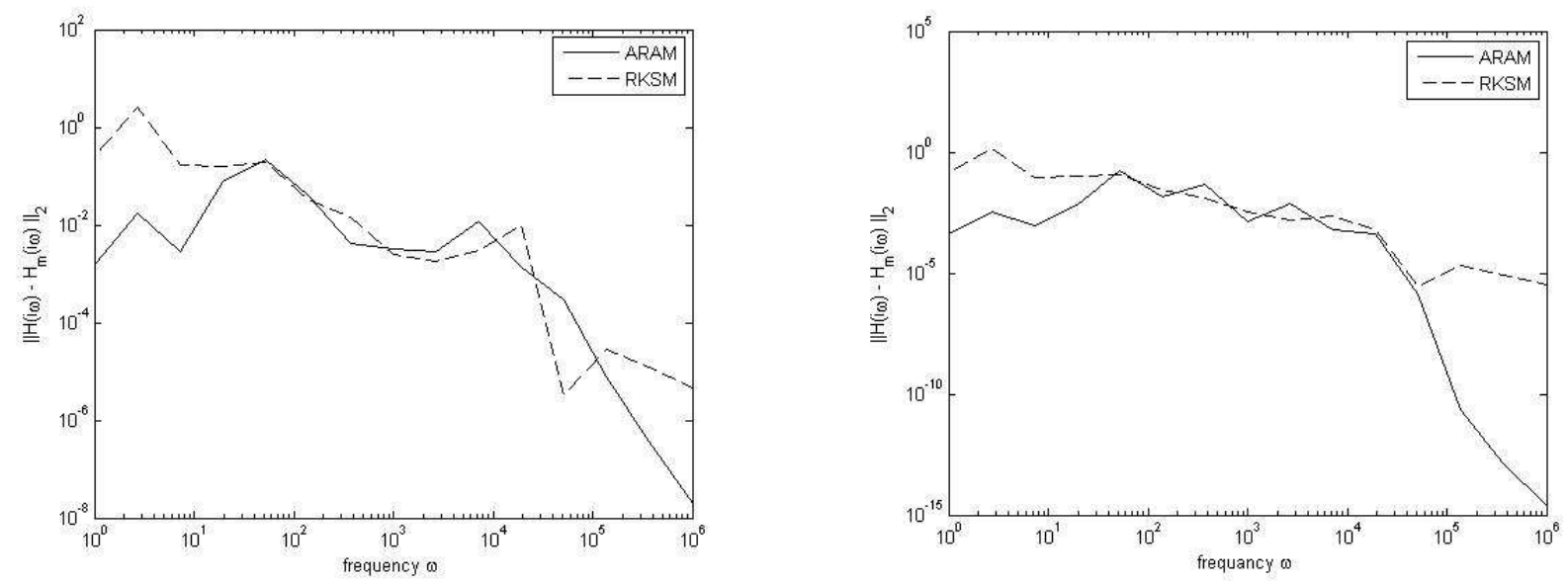

Fig. 3: The CDplayer model . Comparison of ARAM and RKSM. The error $\sigma_{\max }\left(H(i \omega)-H_{m}(i \omega)\right)$ for $\omega \in\left[1,10^{6}\right]$ with $m=8$ (left) and $m=16$ (right).

we used the beam model from Table 1 and we set $m=5$ and $p=3$. The plots in Figure 4 show the original system $\sigma_{\max }(H(i \omega))$ and its approximation $\sigma_{\max }\left(H_{m}(i \omega)\right)$ (left plot), and the associated exact error $\sigma_{\max }\left(H(i \omega)-H_{m}(i \omega)\right)$ for $\omega \in\left[1,10^{6}\right]$.

\section{Conclusion}

In the present paper, we considered new projection methods for model reduction in large scale linear dynamical systems. The proposed methods are Krylov subspace type methods based on the rational block Arnoldi algorithm. We proposed a new procedure for selecting good parameter shifts needed in the proposed rational algorithm and we also give some new algebraic relations. A modified version of the rational block Arnoldi algorithm was also proposed and new simple Arnoldi-like relations were developed. The numerical results show that the method is very attractive for sparse problems. 

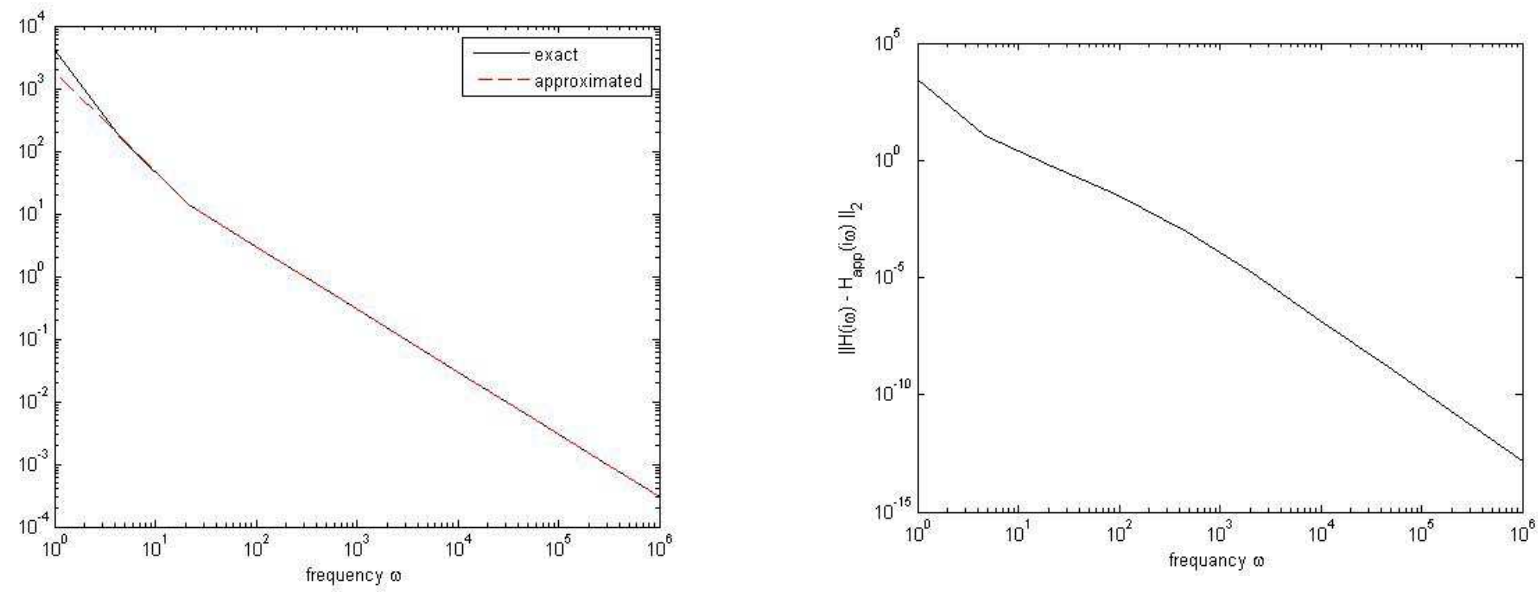

Fig. 4: The beam model: Left: $\|H(i \omega)\|_{2}$ and it's approximation $\left\|H_{m}(i \omega)\right\|_{2}$. Right: the exact error $\left\|H(i \omega)-H_{m}(i \omega)\right\|_{2}$ for $\omega \in\left[1,10^{6}\right]$ with $m=5$ and $p=3$.

\section{References}

[1] Z. Bai, Krylov subspace techniques for reduced-order modeling of large-scale dynamical systems, Appl. Numer. Math. 43 (2002) 944.

[2] Z. Bai, Q. Ye, Error estimation of the Padé approximation of transfer functions via the Lanczos process, Elect. Trans. Numer. Anal. 7 (1998) 1-17.

[3] P. Benner, R.C. Li, N. Truhar, On the ADI method for Sylvester equations, J. Comput. Appl. Math., 233 (2009) 1035-1045.

[4] B. N. Datta, Large-Scale Matrix computations in Control, Appl. Numer. Math. 30 (1999) 53-63.

[5] B. N. Datta, Krylov Subspace Methods for Large-Scale Matrix Problems in Control, Future Gener. Comput. Syst. 19(7) (2003) 1253-1263.

[6] V. Druskin, V. Simoncini, Adaptive rational Krylov subspaces for large-scale dynamical systems, Systems Control Lett. 60(8) (2011) 546-560.

[7] V. Druskin, C. Lieberman, M. Zaslavsky, On adaptive choice of shifts in rational Krylov subspace reduction of evolutionary problems, SIAM J. Sci. Comput. 32(5) (2010) 2485-2496.

[8] K. Gallivan, E. Grimme, P. Van Dooren, A rational Lanczos algorithm for model reduction, Numer. Alg. 12 (1996) 3363.

[9] K. Glover, All optimal Hankel-norm approximations of linear multivariable systems and their L-infinity error bounds. Inter. J. Cont. 39 (1984) 1115-1193.

[10] K. Glover, D. J. N. Limebeer, J. C. Doyle, E. M. Kasenally, M. G. Safonov, A characterisation of all solutions to the four block general distance problem, SIAM J. Control Optim., 29:283-324, (1991).

[11] E. Grimme, Krylov projection methods for model reduction, Ph.D. Thesis, The University of Illinois at Urbana-Champaign. (1997).

[12] E. Grimme, D. Sorensen and P. Van Dooren, Model reduction of state space systems via an implicitly restarted Lanczos method, Numer. Alg. 12 (1996) 1-32.

[13] S. Gugercin, A.C. Antoulas, C. Beattie, $\mathscr{H}_{2}$ model reduction for large-scale linear dynamical systems, SIAM J. Matrix Anal. Appl. 30 (2008) 609-638.

[14] M. Heyouni, K. Jbilou, An extended block Arnoldi algorithm for large-scale solutions of the continuous-time algebraic Riccati equation, Elect. Trans. Num. Anal., 33(2009) 53-62.

[15] M. Heyouni, K. Jbilou, Matrix Krylov subspace methods for large scale model reduction problems, App. Math. Comput., 181(2006) $1215-1228$. 
[16] A. Bouhamidi, M. Hached and K. Jbilou, A preconditioned block Arnoldi for large Sylvester matrix equations, Numer. Lin. Alg. Appl., 2 (2013) 208-219.

[17] C. Jagels, L. Reichel, The extended Krylov subspace method and orthogonal Laurent polynomials, Lin. Alg. Appl., 431(2009), 441-458.

[18] M. Frangos, I.M. Jaimoukha, Adaptive rational interpolation: Arnoldi and Lanczos-like equations, Eur. J. Control., 14(4) (2008) 342-354.

[19] I. M. Jaimoukha, E. M. Kasenally, Krylov subspace methods for solving large Lyapunov equations, SIAM J. Matrix Anal. Appl., 31(1) (1994) 227-251.

[20] L. Knizhnerman and V. Druskin and M. Zaslavsky, On optimal convergence rate of the rational Krylov subspace reduction for electromagnetic problems in unbounded domains, SIAM J. Numer. Anal., 47(2) (2009) 953-971.

[21] B. C. Moore, Principal component analysis in linear systems: controllability, observability and model reduction, IEEE Trans. Automatic Contr., AC-26 (1981) 17-32.

[22] K. Henrik A. Olsson and A. Ruhe, Axel, Rational Krylov for eigenvalue computation and model order reduction, BIT. 46 (2006) S99-S111.

[23] T. Penzl, LYAPACK A MATLAB toolbox for Large Lyapunov and Riccati Equations, Model Reduction Problem, and Linearquadratic Optimal Control Problems, http://www.tu-chemnitz.de/sfb393/lyapack

[24] T. Penzl, LYAPACK A MATLAB toolbox for Large Lyapunov and Riccati Equations, Model Reduction Problem, and Linearquadratic Optimal Control Problems, http://www.tu-chemnitz.de/sfb393/lyapack/guide.pdf

[25] T. Penzl, A cyclic low-rank Smith method for large sparse Lyapunov equations, SIAM J. Sci. Comput. 21 (1999) $1064-8275$.

[26] A. Ruhe, Rational Krylov sequence methods for eigenvalue computation, Lin. Alg. Appl., 58 (1984) 391-405.

[27] A. Ruhe, The rational Krylov algorithm for nonsymmetric eigenvalue problems. III. Complex shifts for real matrices, BIT. 34(1) (1994) 165-176. Y. Saad, Iterative Methods for Sparse Linear Systems, The PWS Publishing Company. (1996).

[28] Y. Shamash, Stable reduced-order models using Padé type approximations, IEEE. Trans. Automatic Control. AC-19 (1974) 615616.

[29] V. Simoncini, A new iterative method for solving large-scale Lyapunov matrix equations, SIAM J. Sci. Comp., 29(3):1268-1288, 2007.

[30] E.L. Wachspress, Iterative solution of elliptic systems, and applications to the neutron diffusion equations of reactor physics, Prentice-Hall, Inc., Englewood Cliffs, N.J. (1966) xiv+299.

[31] E.L. Wachspress, The ADI minimax problem for complex spectra, Academic Press, Boston, MA in Iterative Methods for Large Linear Systems, (1990) 251-271. 